(C) 2021 Universidad Nacional Autónoma de México, Facultad de Estudios Superiores Zaragoza.

Este es un artículo Open Access bajo la licencia CC BY-NC-ND (http://creativecommons.org/licenses/by-nc-nd/4.0/).

TIP Revista Especializada en Ciencias Químico-Biológicas, 24: 1-9, 2021.

https://doi.org/10.22201/fesz.23958723e.2021.375

\title{
Identificación y Actividad Antioxidante de los Compuestos Fenólicos de Jatropha cardiophylla (Torr.) Müll. Arg.
}

\author{
Marcos Leon-Bejarano, Maribel Ovando-Martínez, \\ Claudia Celeste Molina-Domínguez, Rosario Alejandro Trasviña-Mendoza \\ y Luis Ángel Medina-Juárez* \\ Departamento de Investigaciones Científicas y Tecnológicas de la Universidad de Sonora, \\ Blvd. Luis Donaldo Colosio s/n, Entre Reforma y Sahuaripa. Edificio 7G, Col. Centro, \\ Hermosillo, 83000, Sonora, México. *E-mail: luis.medina@unison.mx
}

\begin{abstract}
RESUMEN
México se considera uno de los países con mayor abundancia y diversidad de especies de Jatropha. Jatropha cardiophylla es una planta endémica de las zonas áridas del noroeste de México, con capacidad de adaptación a altas temperaturas y baja humedad. Hasta el momento no existen reportes sobre la identificación de los compuestos fenólicos y su actividad antioxidante en la parte aérea (hojas-tallos) de esta planta. Por este motivo, el objetivo del estudio fue identificar los compuestos fenólicos presentes en el extracto etanólico de la parte aérea (tallo-hoja) de J. cardiophylla y evaluar su actividad antioxidante. Los resultados mostraron la identificación de ácidos fenólicos, gálico, protocatecuico y cafeico; además de los flavonoides como la epicatequina. Por otro lado, el extracto etanólico de esta especie de Jatropha mostró valores de actividad antioxidante de 296.26 $\pm 1.73,416.63$ \pm 3.53 y $456.71 \pm 2.67$ micromoles de equivalentes trolox por gramo de extracto $(\mu \mathrm{molET} / \mathrm{g})$ por los métodos de inhibición del radical DPPH, poder antioxidante reductor del hierro (FRAP) e inhibición del radical ABTS, respectivamente. De acuerdo con los resultados obtenidos, se establece el potencial de la parte aérea de $J$. cardiophylla como fuente de compuestos fenólicos con actividad antioxidante.
\end{abstract}

Palabras clave: actividad antioxidante, compuestos fenólicos, HPLC-DAD, espectrómetro de masas.

\section{Identification and Antioxidant Activity of the Phenolic Compounds of Jatropha cardiophylla (Torr.) Müll. Arg)}

\begin{abstract}
Mexico is considered one of the countries with the greatest abundance and diversity of Jatropha species. Jatropha cardiophylla is an endemic plant to the arid areas of northwestern Mexico, with adaptability to high temperatures and low humidity. So far there are no reports on the identification of phenolic compounds and their antioxidant activity in the aerial part (leaves-stems) of this plant. For this reason, the aim of the study was to identify the phenolic compounds present in the ethanolic extract of the aerial part (stem-leaf) of $J$. cardiophylla and evaluate its antioxidant activity. The results showed the identification of phenolic acids such as gallic, protocatechuic and caffeic acids; as well to flavonoids such as epicatechin. In addition, the ethanolic extract of this Jatropha showed antioxidant activity values of $296.26 \pm 1.73,416.63 \pm 3.53$ and $456.71 \pm 2.67$ micromols of trolox equivalents per gram of extract ( $\mu \mathrm{molTE} / \mathrm{g}$ ) by the inhibition methods of the radical DPPH, antioxidant power reducing iron (FRAP) and inhibition of radical ABTS, respectively. According to the results, the potential of the aerial part of $J$. cardiophylla is established as a source of phenolic compounds with antioxidant activity.
\end{abstract}

Keywords: antioxidant activity, phenolic compounds, HPLC-DAD, mass spectrometer.

Artículo recibido el 11 de marzo del 2021.

Artículo aceptado el 27 de octubre del 2021. 


\section{INTRODUCCIÓN}

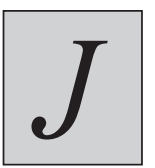

atropha (Euphorbiaceae), pertenece a un género de plantas con 180 especies entre árboles leñosos, matorrales, arbustos e inclusive algunas hierbas, que se encuentran distribuidas ampliamente en regiones tropicales, sub-tropicales, áridas y sub-áridas, principalmente en los continentes de África, Asia y América Central (Zhang et al. 2009; Fresnedo-Ramírez \& Orozco-Ramírez, 2013). Dentro de todas las especies de Jatropha, J. curcas es la más estudiada, principalmente por el alto contenido de aceite en su semilla y su elevado potencial para la producción de biocombustibles (Pandey et al. 2012). Recientemente varias investigaciones se han encaminado a la identificación y obtención de compuestos bioactivos (terpenos, compuestos fenólicos, etc.) con aplicaciones biológicas benéficas para los humanos como antioxidantes, antimicrobianos, antinflamatorios y anti proliferativos, por mencionar algunos (Sabandar, Ahmat, Jaafar \& Sahidin, 2013; Cavalcante, da Conceição Santos \& da Silva Almeida, 2020). Lo anterior, ha generado un creciente interés en el estudio de las plantas de este género.

México se considera uno de los países con mayor abundancia y diversidad de especies de Jatropha (48 especies, 39 de ellas endémicas) algunas distribuidas ampliamente a lo largo del país y otras solamente en regiones específicas. Las especies J. cinerea, J. cordata, J. cuneata, J. malacophylla, y J. cardiophylla representan aproximadamente el $85 \%$ de la diversidad de este género en la región noroeste de México (Fresnedo-Ramírez \& Orozco-Ramírez, 2013). Debido a la riqueza genética de las cinco especies de Jatropha mencionadas anteriormente, estas se encuentran registradas dentro de las Regiones Terrestres Prioritarias de la Comisión Nacional para el Conocimiento y Uso de la Biodiversidad (CONABIO) (Córdova-Télles et al., 2015). Por lo tanto, resulta imprescindible aportar conocimiento sobre los compuestos fitoquímicos de estas especies, que contribuyen a definir un modelo de conservación y aprovechamiento de los recursos naturales.

Los estudios enfocados a la identificación de compuestos bioactivos y evaluación de las actividades biológicas de estas especies son escasos, especialmente para J. cardiophylla. Algunas publicaciones han reportado actividad antifúngica de la parte aérea de $J$. cinerea (Tequida-Meneses, Cortez-Rocha, Rosas-Burgos, López-Sandoval \& Corrales-Maldonado, 2002), y actividad antibacteriana y antifúngica de la parte aérea de $J$. cuneata (Ruiz-Bustos et al. 2009). Sin embargo, estos estudios no identifican a los compuestos responsables de las actividades biológicas. Se han reportado concentraciones de saponinas de 8.2 y $8.9 \mathrm{~g} / \mathrm{kg}$ en semillas de $J$. cardiophylla y $J$. cordata, respectivamente (Gámez-Meza, Alday-Lara, Makkar, Becker \& Medina-Juárez, 2012). Lovio-Fragoso, Gámez-Meza, Molina-Domínguez, Hayano-Kanashiro \& Medina-Juárez, (2017) reportan en J. cinerea un contenido de 200 ppm (partes por millón) de tocoferoles en el aceite de las semillas, así como la presencia de diversos compuestos fenólicos en extractos metanólicos. Por su parte, Alday-Lara (2011) menciona la presencia de ácidos fenólicos (cafeico, clorogénico, sinápico y ferúlico) y flavonoides (miricetina), así como una considerable capacidad antioxidante en extractos metanólicos en las semillas de $J$. cordata y J. cardiophylla. Recientemente, Vega-Ruiz, Hayano-Kanashiro, Gámez-Meza \& Medina-Juárez (2021), detallan la capacidad antioxidante de los extractos metanólicos de tallos y hojas de $J$. cinerea y J. cordata, así como la identificación de compuestos fenólicos como el ácido gálico, el ácido gentísico, la vitexina y el catecol, entre otros.

$J$. cardiophylla es un arbusto perene que alcanza un metro de altura, endémico del margen subtropical oriental del Desierto de Sonora; específicamente desde el norte de Sinaloa, pasando por la región central de Sonora hasta el sur de Arizona. Crece en pendientes arenosas que van desde los 610 a 1,200 metros sobre el nivel del mar, frecuentemente bajo otros arbustos. Sus hojas tienen forma de corazón, alcanzando un tamaño de 1.5 a $7 \mathrm{~cm}$ de largo, son glabras y brillantes, presentan márgenes con dientes redondeados y están agrupadas en brotes cortos o largos. Además, presenta tallos flexibles de color café-rojizo que al cortarlos secretan una sustancia de color rojo, hecho por el que se le conoce a esta planta como sangregado o sangre de Cristo. J. cardiophylla ha sido utilizada por el pueblo Mayo, en el estado de Sonora, para tratar algunos padecimientos. Por ejemplo, la sabia se utiliza para curar la lepra, y el té lo emplean para la cura de la diarrea o llagas en las encías (Kearney, Peebles, Howell \& McClintock, 1960; Yetman \& Van Devender, 2002); información que muestra el potencial bioactivo de la parte aérea de $J$. cardiophylla.

Con base en lo expuesto anteriormente, el objetivo del presente estudio fue la identificación de los compuestos fenólicos presentes en el extracto etanólico de la parte aérea (tallo-hoja) de $J$. cardiophylla y la evaluación de su actividad antioxidante, esto con la finalidad de determinar el potencial antioxidante de esta especie nativa de la región árida del Noroeste de México.

\section{MATERIALES Y MÉTODOS \\ Obtención de la muestra}

Las hojas y tallos de $J$. cardiophylla (Figura 1) se colectaron el mes de agosto de 2018 en cuatro áreas circundantes del municipio de Hermosillo, Sonora, México (área 1: $28^{\circ} 52^{\prime} 7.23^{\prime \prime} \mathrm{N}$ $110^{\circ} 44^{\prime} 8.62^{\prime \prime} \mathrm{O}$, área $2: 29^{\circ} 00^{\prime} 47.8^{\prime \prime} \mathrm{N} 111^{\circ} 02^{\prime} 21.3^{\prime \prime} \mathrm{O}$, área $3: 29^{\circ} 12^{\prime} 19.2^{\prime \prime} \mathrm{N} 111^{\circ} 44^{\prime} 01^{\prime \prime} 20.7 \mathrm{O}$ y área $4: 29^{\circ} 10^{\prime} 59.2^{\prime \prime} \mathrm{N}$ $\left.110^{\circ} 55^{\prime} 51.8^{\prime \prime} \mathrm{O}\right)$. Se seleccionaron 6 individuos sanos por cada área, y se colectaron tallos con abundante hoja obteniendo un peso aproximado de $200 \mathrm{~g}$ por individuo, los cuales se almacenaron en bolsas de papel de $50 \times 20 \mathrm{~cm}$.

\section{Procesamiento de la muestra}

Las hojas y los tallos fueron secados individualmente en un horno al vacío a $60{ }^{\circ} \mathrm{C}$ y posteriormente pulverizados con 


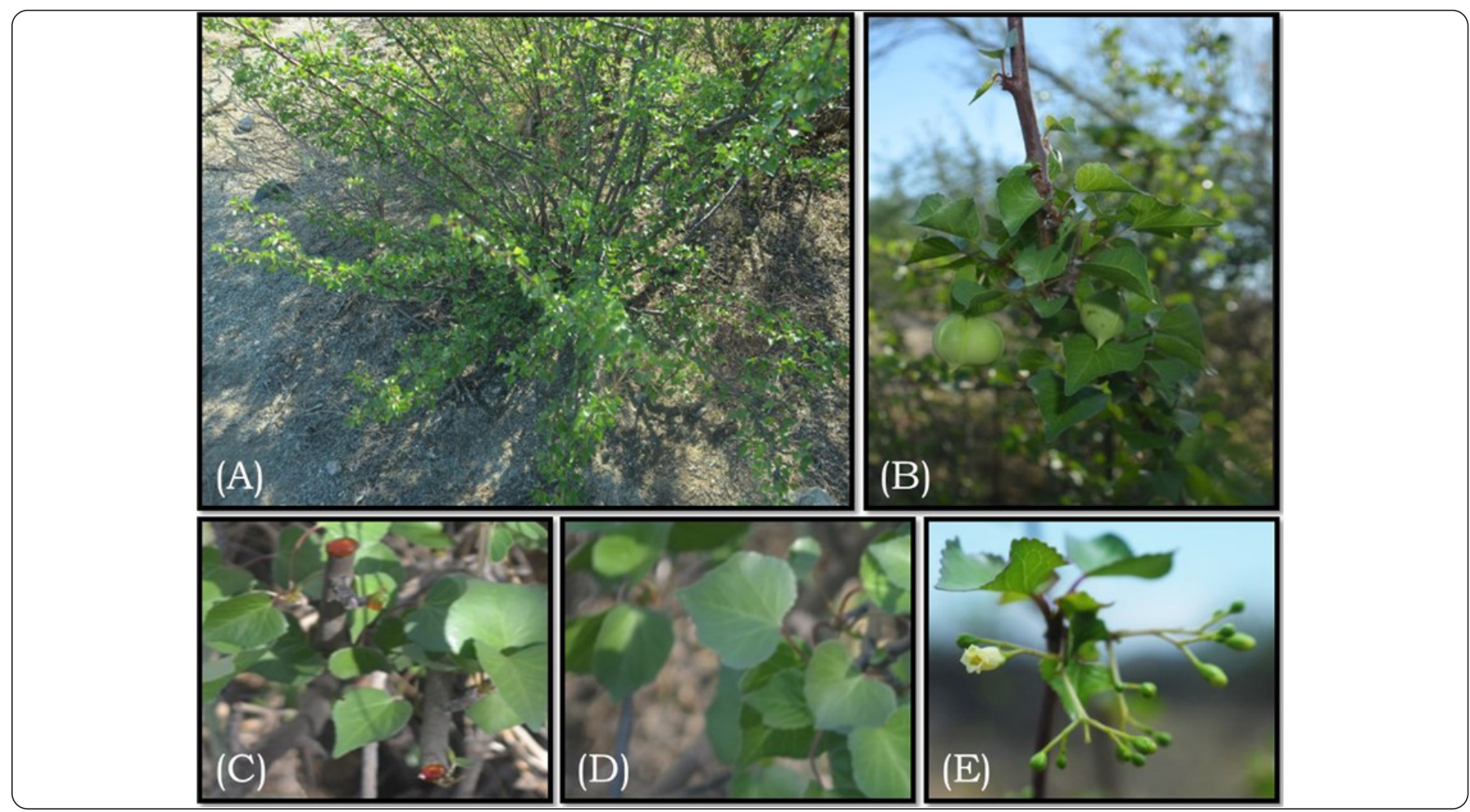

Figura 1. Jatropha cardiophylla. Planta completa (A), tallo con hojas y semillas (B), secreción similar a sangre en el tallo (C), hojas similares a un corazón (D) y flores (E). Fotos tomadas por Marcos Leon-Bejarano y Rosario Alejandro Trasviña-Mendoza.

ayuda de una licuadora industrial. Las muestras pulverizadas se llevaron a un tamaño de partícula menor a $1 \mathrm{~mm}$ mediante un molino de martillo (Thomas-Wiley Laboratory Mill Model 4). Después, la muestra se mantuvo en un frasco ámbar a $4{ }^{\circ} \mathrm{C}$ hasta su utilización.

\section{Preparación de los extractos}

Los extractos etanólicos se obtuvieron siguiendo la metodología descrita por Xu et al. (2016), con modificaciones. La muestra se mezcló vigorosamente con la solución de etanol al $60 \%$ en una relación de 1/30 (p/v). La mezcla se sometió a sonicación por 15 min (Branson Sonicador, model 1510, USA) y se centrifugaron (Centrifuge IEC CL3 IR, Thermo Electron Industries SAS, Chaâteau-Gontier, Mayenne, France) a 5,000 g por 15 min a $4{ }^{\circ} \mathrm{C}$. El sobrenadante se filtró con papel Whatman \#4. Este proceso de extracción se repitió nuevamente y el extracto final se llevó a sequedad en un rotavapor (Labconco, USA) y se liofilizaron (FreeZone 4.5, Labconco, Kansas City, MO, USA). El extracto resultante se mantuvo en un frasco ámbar a $4{ }^{\circ} \mathrm{C}$ hasta su análisis. Para los ensayos de actividad antioxidante, el extracto se disolvió en una solución de etanol al $60 \%$.

\section{Análisis de HPLC-DAD}

Para la identificación y cuantificación de los compuestos fenólicos los extractos se sometieron a un proceso de hidrólisis ácida (Hilbig et al. 2018). Se dejaron $150 \mathrm{mg}$ de cada extracto en $12 \mathrm{~mL}$ de ácido clorhídrico $2 \mathrm{M}$ por $2 \mathrm{~h}$ a $80^{\circ} \mathrm{C}$ con agitación constante. La mezcla se dejó enfriar y se ajustó a un $\mathrm{pH}$ de 2 con una solución de $\mathrm{NaOH}$. Posteriormente, se realizó una extracción de $18 \mathrm{~mL}$ de éter etílico por tres ocasiones, las fases orgánicas fueron recolectadas y combinadas. Finalmente, el solvente se eliminó con un rotavapor a $28{ }^{\circ} \mathrm{C}$ y el extracto resultante se liofilizó.

El extracto hidrolizado se resuspendió en metanol puro y se filtró con microfiltro de nylon $(0.20 \mu \mathrm{L})$ en un vial para su inyección $(50 \mu \mathrm{L})$ en el equipo de HPLC-DAD (Agilent 1,200, Santa Clara, California) equipado con una columna C18 ( $25 \mathrm{~cm}$ x $4 \mathrm{~mm}$ x $5 \mu \mathrm{m}$ ) (Supelcosil, Sigma). La fase móvil consistió en agua acidificada al 1\% con ácido acético (A) y metanol (B) con el siguiente gradiente de elución de $1 \mathrm{~mL} / \mathrm{min}$ : $90 \%$ de A de 0 a 27 min, de 90 a $60 \%$ de A en 28 min, $60 \%$ de A por $5 \mathrm{~min}$, de $60 \%$ a $56 \%$ de A en $2 \mathrm{~min}, 56 \%$ de A por $8 \mathrm{~min}$, de $56 \%$ a $90 \%$ de A en $1 \mathrm{~min}$ y $90 \%$ de A por $4 \mathrm{~min}$. (Nour, Trandafir \& Cosmulescu, 2013). Los cromatogramas fueron obtenidos a una longitud de onda de 254, 278, 300 y $360 \mathrm{~nm}$. Posteriormente la identificación de los compuestos fenólicos en la muestra, se realizó comparándolos con los tiempos de retención de los estándares puros (Sigma-Aldrich). Estos compuestos fueron cuantificados utilizando curvas patrón de los estándares correspondientes. Los resultados se reportaron como mg del compuesto fenólico por gramos 
de peso seco de la muestra (mg/g PS). El análisis se realizó por triplicado.

Confirmación de compuestos fenólicos por ESI-IT-MS-MS Para la confirmación de los compuestos fenólicos de los extractos etanólicos de la parte aérea (hojas y tallos) de Jatropha cardiophylla, se realizaron inyecciones de $10 \mu \mathrm{g} / \mathrm{mL}$ por infusión directa a un espectrómetro de masas con electro spray y trampa de iones (FIA-ESI-IT-MS/MS, Varian $500 \mathrm{MS}$, Santa Clara, CA, EUA). El análisis fue por ionización negativa, utilizando nitrógeno, como gas nebulizador y helio como gas de colisión. El intervalo de análisis fue de 100-2,000 m/z. Los iones negativos de los compuestos fenólicos se obtuvieron bajo un flujo de infusión de $10 \mu \mathrm{g} / \mathrm{mL}$, voltaje de $-17 \mathrm{kV}$, una temperatura de capilar de $350{ }^{\circ} \mathrm{C}$ y un flujo de gas auxiliar de $1 \mathrm{~mL} / \mathrm{min}$. La manipulación del extracto se realizó en condiciones de oscuridad (Vega-Ruiz et al., 2021).

\section{Actividad antioxidante por inhibición del radical ABTS}

El radical catiónico ácido 2,2'-azino-bis (3-etilbenzotiazolín6-sulfónico) ABTS •+ fue preparado haciendo reaccionar una solución madre de ABTS $(7 \mathrm{mM})$ con persulfato de potasio $2.45 \mathrm{mM}$ (concentración final) y dejando la mezcla reposar en la oscuridad a temperatura ambiente durante $16 \mathrm{~h}$ antes de su uso. La solución de trabajo de ABTS $\bullet+$ se obtuvo diluyendo $500 \mu \mathrm{L}$ de la solución madre en $35 \mathrm{~mL}$ de etanol, hasta una absorbancia de $0.7 \pm 0.02 \mathrm{~nm}$ a $750 \mathrm{~nm}$. (Re et al. 1999; Miller, Rice-Evans, Davies, Gopinathan \& Milner, 1993). En un tubo de ensaye de vidrio se mezclaron $20 \mu \mathrm{L}$ del extracto con $2 \mathrm{~mL}$ del ABTS • + previamente preparado, y se dejó incubar por $5 \mathrm{~min}$. Posteriormente, se colocaron $250 \mu \mathrm{L}$ en una microplaca de 96 pozos y se realizó la lectura de absorbancia a $750 \mathrm{~nm}$ utilizando un lector de microplacas (Varioskan LUX Multimode Microplate Reader, Thermo Scientific, Vantaa, Finland). Los resultados fueron expresados como $\mu \mathrm{molET} / \mathrm{g}$, utilizando una curva estándar de trolox. El ensayo se realizó por triplicado.

\section{Actividad antioxidante por inhibición del radical DPPH}

El radical DPPH 'se preparó mezclando el reactivo DPPH (2,2-difenil-1-picrilhidrazilo) en etanol $(50 \mathrm{mM})$. La absorbancia de la solución de trabajo se ajustó a $0.70 \pm 0.02$ a $516 \mathrm{~nm}$. El experimento se llevó a cabo en microplacas de 96 pocillos donde se agregaron $20 \mu \mathrm{L}$ del extracto a cada pozo y $180 \mu \mathrm{L}$ de solución de trabajo DPPH. Luego, la placa se incubó en la oscuridad durante $30 \mathrm{~min}$ a temperatura ambiente y se realizó la lectura a $516 \mathrm{~nm}$. Los resultados se expresaron como $\mu \mathrm{molET} / \mathrm{g}$ ), utilizando una curva estándar de trolox. El ensayo se realizó por triplicado (Brand-Williams W., Cuvelier $\&$ Berset, 1995).

\section{Poder Antioxidante Reductor del Hierro (FRAP)}

El ensayo FRAP se realizó de acuerdo con lo descrito por Benzie \& Strain (1996) con modificaciones. El reactivo FRAP se preparó mezclando una solución amortiguadora de acetato de sodio (pH 3.6, 0.3 M), una solución $10 \mathrm{mM}$ de 2,4,6-tri (2-piridil)-s-triazina (TPTZ) en $\mathrm{HCl} 40 \mathrm{mM}$ y una solución acuosa de $\mathrm{FeCl}_{3} 20 \mathrm{mM}$ en una relación 10:1:1, respectivamente. En una microplaca de 96 pozos se colocaron $20 \mu \mathrm{L}$ del extracto y $280 \mu \mathrm{L}$ del reactivo FRAP, se dejó incubar por $30 \mathrm{~min}$ y se realizó la lectura de absorbancia a $750 \mathrm{~nm}$. Los resultados se expresaron como $\mu \mathrm{molET} / \mathrm{g}$, utilizando una curva estándar de trolox. El ensayo se realizó por triplicado.

\section{RESULTADOS Y DISCUSIÓN}

Perfil de compuestos fenólicos en los extractos etanólicos de la parte aérea de $J$. cardiophylla

Para identificar en el HPLC-DAD a los compuestos fenólicos del extracto etanólico de la parte aérea de $J$. cardiophylla, se realizó un proceso de hidrólisis para la obtención en forma libre de estos compuestos. Con el análisis de HPLC-DAD se identificaron inicialmente 13 distintos compuestos fenólicos, de los que se confirmó por ESI-IT-MS-MS la presencia de 10, cuyas concentraciones se muestran en la Tabla I. Dentro de los 10 compuestos fenólicos, el ácido gálico presentó la mayor concentración, seguido de la epicatequina, el ácido protocatecuico y el ácido cafeico. Anteriormente, AldayLara (2011) encontró miricetina como compuesto fenólico principal en los extractos metanólicos de la semilla de $J$. cardiophylla, además de la presencia de ácidos fenólicos (ferúlico $>$ clorogénico $>$ sinápico $>$ cafeico). En comparación con otras especies de la región, Alday-Lara (2011) reportó ácido sinápico como compuesto mayoritario en la semilla de J. cordata. Por otro lado, el ácido cafeico fue el compuesto predominante en los extractos metanólicos de la semilla de $J$. cinerea (Lovio-Fragoso et al. 2017). Sin embargo, en ese estudio se analizó cualitativamente el extracto por medio de la espectrometría de masas, identificando 10 compuestos adicionales como el ácido gálico, el sinápico, el protocatecuico, el ferúlico, la epicatequina y la quercetina, que se identificaron y cuantificaron. Recientemente Vega-Ruizet al (2021), reportaron la composición fenólica de extractos metanólicos de la parte aérea de $J$. cinerea y $J$. cordata. De acuerdo con los autores, el compuesto de mayor concentración en las hojas de ambas especies fue el ácido gentísico; mientras que, la epicatequina y la quercetina, fueron los compuestos mayoritarios en los tallos de $J$. cinerea y $J$. cordata, respectivamente. De igual forma, los autores destacan las diferencias tanto en la composición como en la concentración de los compuestos entre los tejidos estudiados (hoja y tallo), que concuerda con lo mencionado anteriormente. En términos generales las especies de Jatropha de la zona cercana al municipio de Hermosillo, Sonora comparten un perfil de compuestos fenólicos similar. Sin embargo, las diferencias entre los perfiles, podría interferir en la capacidad antioxidante de cada una de las especies.

Por otro lado, y como se muestra en la Figura 2, la composición del extracto no sólo cuenta con los compuestos identificados y cuantificados, sino que se pueden observar una variedad 
Tabla I. Compuestos fenólicos identificados en el extracto etanólico de la parte aérea de Jatropha cardiophylla.

\begin{tabular}{|l|c|c|c|}
\hline \multicolumn{1}{|c|}{ Compuesto fenólico } & Longitud de onda (nm) & Tiempo de Retención (min) & mg/gPS \\
\hline 1 Ácido gálico & 278 & 4.08 & $116.86 \pm 4.18^{\mathrm{a}}$ \\
\hline 2 Ácido protocatecuico & 254 & 7.33 & $14.94 \pm 1.50^{\mathrm{d}}$ \\
\hline 3 Ácido hidroxibenzoico & 254 & 12.94 & $2.38 \pm 0.10^{\mathrm{ghi}}$ \\
\hline 4 Catequina & 278 & 14.77 & $0.71 \pm 0.33^{\mathrm{i}}$ \\
\hline 5 Ácido cafeico & 300 & 21.81 & $13.01 \pm 2.12^{\mathrm{d}}$ \\
\hline 6 Epicatequina & 278 & 37.68 & $23.08 \pm 3.21^{\mathrm{c}}$ \\
\hline 7 Ácido $p$-cumárico & 300 & 38.64 & $9.80 \pm 0.67^{\mathrm{e}}$ \\
\hline 8 Ácido ferúlico & 300 & 44.88 & $2.25 \pm 0.16^{\text {ghi }}$ \\
\hline 9 Exicatequina galato & 278 & 45.91 & $2.79 \pm 0.04^{\mathrm{gh}}$ \\
\hline 10 Ácido sinápico & 300 & 47.45 & $0.90 \pm 0.34^{\mathrm{hi}}$ \\
\hline
\end{tabular}

Valores \pm desviación estándar $(\mathrm{n}=3)$. Letras diferentes indican diferencias significativas $(\mathrm{p}<0.05)$. $(\mathrm{mg} / \mathrm{g}$ PS $)=$ mg del compuesto fenólico por gramos de peso seco de la muestra.

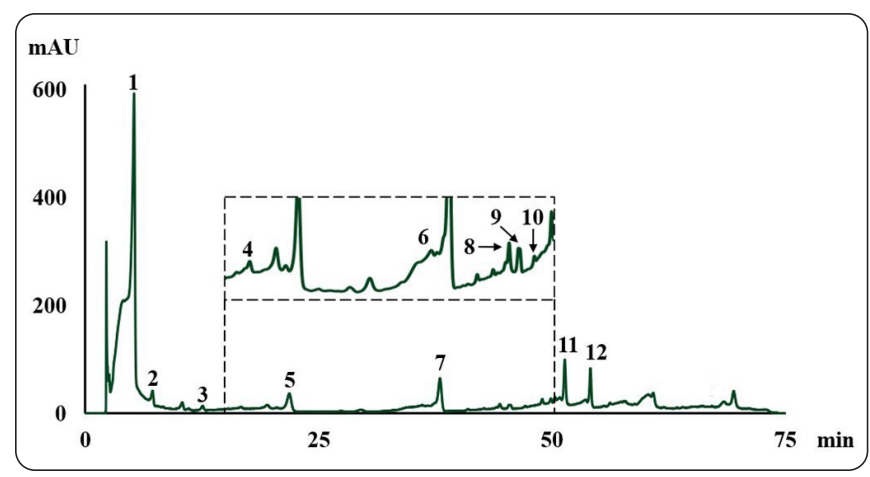

Figura 2. Cromatograma del extracto de Jatropha cardiophylla a $278 \mathrm{~nm}$. Los números corresponden al compuesto fenólico listado en la Tabla I.

de picos, lo que nos indica la presencia de otros compuestos que también podrían estar involucrados en la capacidad antioxidante de los extractos. Por este motivo, se incluyó en el presente trabajo la confirmación de los compuestos fenólicos por ESI-IT-MS-MS, al igual que lo realizado por Lovio-Fragoso et al (2017) y Vega-Ruiz et al (2021) en otras especies de la región.

Confirmación de compuestos fenólicos por ESI-IT-MS-MS Una vez realizado el análisis de los compuestos fenólicos presentes en el extracto etanólico de la parte aérea (hojas y tallos) de Jatropha cardiophylla mediante HPLC-DAD, se llevó a cabo la comprobación de estos, por infusión directa y por espectrometría de masas (Figura 3). La identificación de cada compuesto fenólico presente en los extractos se realizó mediante fragmentación de segundo orden (MS / MS). La confirmación se realizó comparando los iones fragmentados con patrones puros

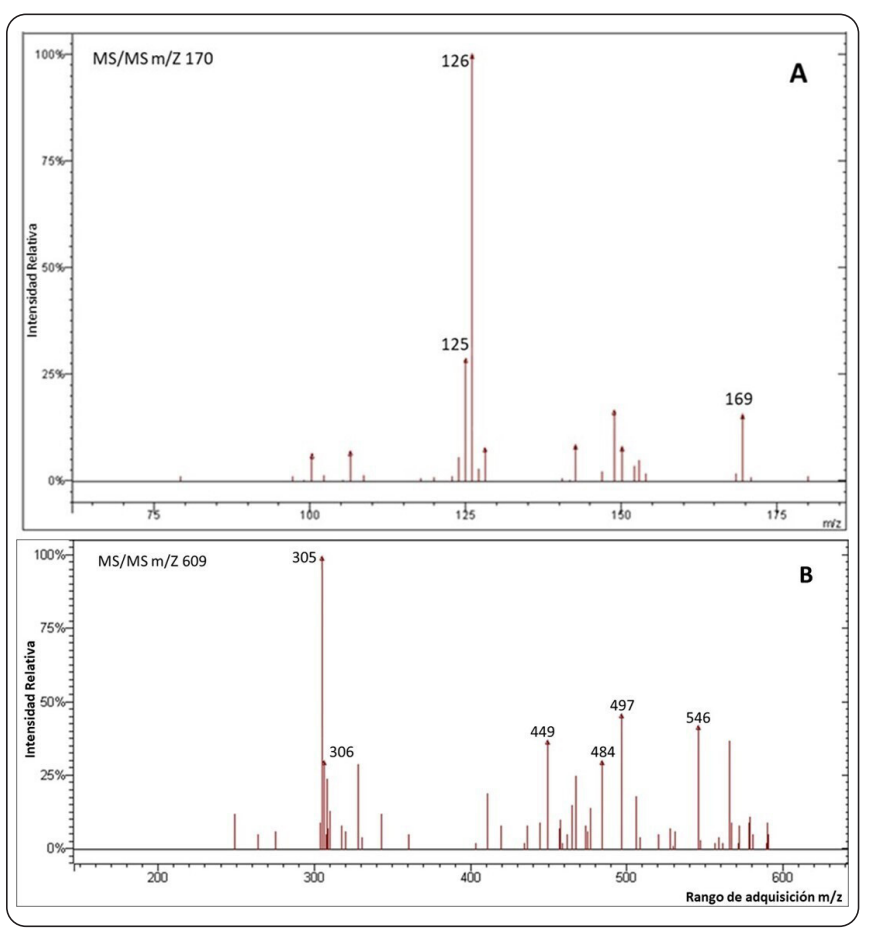

Figura 3. Ion fragmentado de dos compuestos fenólicos encontrados en la parte aérea de $J$. Cardiophylla mediante espectrometría de masas (ESI-IT-MS / MS). (A) Ácido gálico. (B) Dímero de [Epi] galocatequina.

(Tabla II). Entre los compuestos confirmados se detectaron compuestos libres y otros como dímeros o trímeros. En el caso de vitexina, rutina y quercitina, no se encontró evidencia en el espectro de masas, de su presencia en el extracto etanólico de la parte aérea de $J$. cardiophylla. 
Tabla II. Confirmación de los compuestos fenólicos por ESI-IT-MS-MS, presentes en el extracto etanólico de la parte aérea de Jatropha cardiophylla.

\begin{tabular}{|l|c|c|l|}
\hline \multicolumn{1}{|c|}{ Compuesto } & Peso molecular & $\begin{array}{c}\text { Ion Precursor } \\
{[\mathbf{M}-\mathbf{H}]-\boldsymbol{m} / \boldsymbol{z}}\end{array}$ & \multicolumn{1}{|c|}{$\begin{array}{c}\text { Fragmento de iones ESI-MS } \\
\text { (Intensidad relativa) }\end{array}$} \\
\hline 1 Ácido gálico & 170 & 169 & $125(28), 126(100)$ \\
\hline 2 Ácido protocatecuico & 154 & 153 & $109(100)$ \\
\hline 3 Ácido hidroxibenzoico & 138 & 137 & $92(100), 93(26), 136(50), 137(3)$ \\
\hline 4 Catequina & 290 & 289 & $245(7), 227(18), 205(31), 203(100), 179(5)$ \\
\hline 5 Ácido cafeico & 180 & 179 & $135(100), 179(15)$ \\
\hline 6 Epicatequina & 290 & 289 & $203(100), 205(31), 227(18), 245(7)$ \\
\hline 7 Ácido p-cumárico & 164 & 163 & $134(100), 119(65)$ \\
\hline 8 Ácido ferúlico & 194 & 193 & $111(100), 121(8), 137(11), 175(3)$ \\
\hline 9 Ácido sinápico & 224 & 223 & $208(100), 164(33), 179(47)$ \\
\hline 10 [Epi]galocatequina-[Epi]catequina & 594 & 593 & $289(100), 305(2), 407(4), 425(7)$ \\
\hline 11 Dímero de [Epi]galocatequina & 610 & 609 & $305(100), 484(30)$ \\
\hline 12 Ácido elágico & 302 & 301 & $185(100), 257(46)$ \\
\hline 13 Ácido metilelágico & 316 & 315 & $185(100), 300(2)$ \\
\hline 14 Ácido dimetilelágico & 330 & 329 & $301(100), 314(8), 185(100)$ \\
\hline 15 Laricitrina & 332 & 331 & $169(3), 288(57), 315(47), 185(100)$ \\
\hline 16 Ácido Vań́lico & 168 & 167 & $123(100), 110(72)$ \\
\hline 27 Aldehído Protocatecuico & 138 & 137 & $109(100), 92(95), 135(83)$ \\
\hline
\end{tabular}

Actividad antioxidante de los extractos etanólicos de la parte aérea de $J$. cardiophylla

Comúnmente la actividad antioxidante de los extractos de plantas es evaluada mediante diversos métodos basados en los principales mecanismos antioxidantes, como transferencia de electrones (SET) y transferencia de átomo de hidrógeno (HAT). En este contexto, el método de FRAP se basa en la reducción del ion $\mathrm{Fe}^{3+} \mathrm{a} \mathrm{Fe}^{2+}$ mediante el mecanismo SET. Por otro lado, los métodos ABTS· + y DPPH· responden a ambos mecanismos, HAT y SET. Entre las principales diferencias de ambos métodos podemos mencionar que el método de $\mathrm{ABTS}++$ presenta mayor facilidad de interacción tanto con los compuestos lipofílicos como con los hidrofílicos, y que el método de DPPH· tiene la limitante estérica de reaccionar con moléculas de gran tamaño, aunque también se considera más selectivo que su contraparte $\mathrm{ABTS} \cdot+$, ya que este último puede reaccionar con moléculas que poseen un solo grupo hidroxilo (Holtz, 2009; Cerretani \& Bendini, 2010).

En este estudio, los extractos presentaron valores de $456.71 \pm$ $2.67,416.63 \pm 3.53$ y $296.26 \pm 1.73 \mu \mathrm{molET} / \mathrm{g}$, en los métodos de ABTS + , FRAP y DPPH', respectivamente. (Tabla III). De acuerdo con lo ya mencionado se infiere que los compuestos presentes en el extracto actúan principalmente por el mecanismo SET, indicando una mayor abundancia de compuestos de mayor tamaño molecular y/o la posible presencia de compuestos lipofílicos. En comparación con otros estudios, los extractos del presente trabajo mostraron un porcentaje de inhibición del radical DPPH ( $64 \%)$ similar a los extractos metanólicos de la semilla de $J$. cardiophylla ( 48-72\%) y mayor a los extractos de la semilla de $J$. cordata, ambas recolectadas en el estado de Sonora (Alday-Lara 2011). Por su parte, Wong-Paz

Tabla III. Actividad antioxidante de los extractos etanólicos de la parte aérea de Jatropha cardiophylla.

\begin{tabular}{|l|c|c|}
\hline & MmolET/g & \% de Inhibición \\
\hline Inhibición del radical ABTS & $456.71 \pm 2.67^{\mathrm{a}}$ & $34.29 \pm 0.24$ \\
\hline Inhibición del radical DPPH & $296.26 \pm 1.73^{\mathrm{c}}$ & $64.09 \pm 0.23$ \\
\hline FRAP & $416.63 \pm 3.53^{\mathrm{b}}$ & - \\
\hline
\end{tabular}

Valores \pm desviación estándar $(\mathrm{n}=3)$. Letras diferentes indican diferencias significativas $(\mathrm{p}<0.05)$. 
et al. (2015), reportaron porcentajes de inhibición de $\sim 15 \%$ para DPPH y $\sim 24 \%$ para ABTS, en los extractos etanólicos del tallo-raíz de $J$. dioica procedente del estado de Coahuila. Diversos extractos del tallo y raíz de $J$. dioica recolectada en una zona semiárida del estado Hidalgo, México presentaron porcentajes de inhibición entre $17.7-44.3 \%$ para DPPH y 29.1-49.9\% para ABTS (Gutiérrez-Tlahque et al. 2019). En estos estudios los valores fueron inferiores a los del presente trabajo, con excepción de los extractos de raíz de $J$. dioica con un porcentaje de inhibición mayor. Recientemente, en un estudio realizado por Vega-Ruiz et al. (2021), en los extractos metanólicos de las hojas y los tallos de $J$. cinerea se vio que el porcentaje de inhibición fue bajo en comparación con el de este trabajo por ambos métodos $(<15 \%$ para ABTS y $<23 \%$ para DPPH); de manera contraria, los extractos de las hojas y los tallos de $J$. cordata tuvieron valores mayores a los de nuestro estudio (47-54\% para ABTS y $73-77 \%$ para DPPH). Es así como los resultados demuestran el potencial antioxidante de $J$. cardiophylla al ser comparable con otras especies de la región.

El potencial antioxidante observado en los extractos podría estar relacionado con los principales compuestos fenólicos del extracto. El ácido gálico (Figura 4) se encuentra en los tejidos



Figura 4. Estructura química de los compuestos fenólicos identificados por ESI-IT-MS-MS, presentes en el extracto etanólico de la parte aérea de Jatropha cardiophylla. Figuras construidas por Claudia Celeste Molina-Domínguez. 
vegetales en forma de éster (ésteres con glucósidos, polioles y fenoles). Sin embargo, puede estar en forma libre, derivado de un proceso de hidrólisis, en algunos alimentos como las frutas, verduras y bebidas. En forma libre, el ácido gálico es un compuesto fenólico de bajo peso molecular constituido por tres grupos hidroxilo y un ácido carboxílico unidos a un anillo aromático con una alta capacidad antioxidante, debido principalmente a su conformación estructural. Además, se ha publicado que este compuesto presenta efecto anticancerígeno, antiinflamatorio y antiviral (Badhani, Sharma \& Kakkar, 2015). Por otro lado, la epicatequina (Figura 4) es un flavonoide presente como $O$-glucósido en los tejidos vegetales (principalmente en las hojas) con un total de cinco grupos hidroxilos que contribuyen notablemente en su actividad antioxidante (Terahara, 2015).

\section{CONCLUSIONES}

Los resultados de este trabajo muestran que la parte aérea (hojas y tallos) de Jatropha cardiophylla contiene compuestos fenólicos entre cuyos ácidos destacan el gálico, el protocatecuico y el cafeico además de flavonoides como la epicatequina. Por otro lado, estos extractos mostraron actividad antioxidante similar a lo reportado para otras especies de Jatropha nativas de las zonas áridas del Noroeste de México. Esto muestra además que las hojas y tallos de $J$. cardiophylla podrían tener un uso potencial similar al reportado para otras especies del género Jatropha nativas de México.

\section{Agradecimientos}

Marcos Leon-Bejarano, agradece al Consejo Nacional de Ciencia y Tecnología (CONACYT) por la beca otorgada.

\section{REFERENCIAS}

Alday-Lara, P. P. (2011). Evaluación de las semillas de Jatropha cordata y Jatropha cardiophylla, comofuentes de proteina, compuestos fenólicos y aceite para la obtención de biodiesel. Tesis. (pp. 65-70). Hermosillo, Sonora México: Universidad de Sonora.

Badhani, B., Sharma, N. \& Kakkar, R. (2015). Gallic acid: a versatile antioxidant with promising therapeutic and industrial applications. Royal Society of Chemistry Advances, 5, 27540-27557. https://doi.org/10.1039/ C5RA01911G

Benzie, I. F. F. \& Strain, J. J. (1996). The Ferric Reducing Ability of Plasma (FRAP) as a Measure of "Antioxidant Power": The FRAP Assay. Analytical Biochemistry, 239(1), 70-76. https://doi.org/10.1006/abio.1996.0292.

Brand-Williams, W., Cuvelier, M. E. \& Berset. C. (1995). Use of a Free Radical Method to Evaluate Antioxidant Activity. LWT-Food Science and Technology, 28(1), 25-30. https:// doi.org/10.1016/S0023-6438(95)80008-5.

Cavalcante, N. B., da Conceição Santos, A. D. \& da Silva Almeida, J. R. G. (2020). The genus Jatropha (Euphorbiaceae): A review on secondary chemical metabolites and biological aspects. Chemico-Biological Interactions, 318, 108976. https://doi.org/10.1016/j. cbi.2020.108976.

Cerretani, L. \& Bendini, A. (2010). Chapter 67 - Rapid Assays to Evaluate the Antioxidant Capacity of Phenols in Virgin Olive Oil, Editors: Preedy, V. R. \& Watson, R. R., Olives and Olive Oil in Health and Disease Prevention, Academic Press, Pages 625-635. https://doi.org/10.1016/B978-0-12374420-3.00067-X.

Córdova-Télles, L., Bautista-Ramírez, E., ZamarripaColmenero, A., Rivera Lorca, J. A., Pérez-Vázquez, A., Sánchez-Sanchez., O. M., Martínez-Herrera, J. \& CuevasSánchez, J. A. (2015). Diagnóstico y Plan Estratégico de la Red Jatropha spp. En México. SNICS, SINAREFI, México. 116p.

Fresnedo-Ramírez, J. \& Orozco-Ramírez, Q. (2013). Diversity and distribution of genus Jatropha in México. Genetic Resources and Crop Evolution, 60(3), 1087-1104. https:// doi.org/10.1007/s10722-012-9906-7.

Gámez-Meza, N., Alday-Lara, P. P., Makkar, H. P., Becker, K. \& Medina-Juárez, L. A. (2012). Chemical characterisation of kernels, kernel meals and oils from Jatropha cordata and Jatropha cardiophylla seeds. Journal of the Science of Food and Agriculture, 93(7), 1706-1710. https://doi. org/10.1002/jsfa.5955

Gutiérrez-Tlahque, J., Aguirre-Mancilla, C. L., López-Palestina, C., Sánchez-Fernández, R. E., Hernández-Fuentes, A. D. \& Torres-Valencia, J. M. (2019). Constituents, antioxidant and antifungal properties of Jatropha dioica var. Dioica. Natural Product Communications, 14(5), 1-5. https://doi.org/10.1177/1934578X19852433.

Hilbig, J., de Britto Policarpi, P., de Souza Grinevicius, V. M. A., Santos Mota, N. S. R. S., Toaldo, I. M., Bordignon Luiz, M. T., Curi Pedrosa R. \& Block, J. M. (2018). Aqueous extract from pecan nut [Carya illinoinensis (Wangenh) C. Koch] shell show activity against breast cancer cell line MCF-7 and Ehrlich ascites tumor in Balb-C mice. Journal of Ethnopharmacology, 211, 256-266. https://doi. org/10.1016/j.jep.2017.08.012.

Holtz, R. W. (2009). Chapter 13-In Vitro Methods to Screen Materials for Anti-aging Effects. (Ed) Nava Dayan, In Personal Care \& Cosmetic Technology, Skin Aging Handbook, William Andrew Publishing 329-362. https:// doi.org/10.1016/B978-0-8155-1584-5.50017-X.

Kearney, T. H., Peebles, R. H., Howell, H. T. \& McClintock, E. (1960). Arizona flora. Univ of California Press.

Lovio-Fragoso, J. P., Gámez-Meza, N., Molina-Domínguez, C. C., Hayano-Kanashiro, C. \& Medina-Juárez, L. A. (2017). Physical characteristics and chemical constituents of Jatropha cinerea seeds. RISG-Rivista Italiana Sostanze Grasse, 94(3), 169-174.

Miller, N. J., Rice-Evans, C., Davies, M. J., Gopinathan, V. \& Milner, A. (1993). A Novel Method for Measuring Antioxidant Capacity and its Application to Monitoring 
the Antioxidant Status in Premature Neonates. Clinical Science, 84 (4), 407-412. DOI: https://doi.org/10.1042/ $\operatorname{cs} 0840407$

Nour, V., Trandafir, I. \& Cosmulescu, S. (2013). HPLC determination of phenolic acids, flavonoids and juglone in walnut leaves. Journal of chromatographic science, 51(9), 883-890. https://doi.org/10.1093/chromsci/bms180.

Pandey, V. C., Singh, K., Singh, J. S., Kumar, A., Singh, B. \& Singh, R. P. (2012). Jatropha curcas: A potential biofuel plant for sustainable environmental development. Renewable and Sustainable Energy Reviews, 16(5), 28702883. https://doi.org/10.1016/j.rser.2012.02.004.

Re, R., Pellegrini, N., Proteggente, A., Pannala, A., Yang, M. \& Rice-Evans C. (1999). Antioxidant Activity Applying an Improved ABTS Radical Cation Decolorization Assay. Free Radical Biology and Medicine, 26(9), 1231-1237. https://doi.org/10.1016/S0891-5849(98)00315-3.

Ruiz-Bustos, E., Velázquez, C., Garibay-Escobar,A., García,Z., Plascencia-Jatomea, M., Cortez-Rocha, M. O., HernándezMartínez, J. \& Robles-Zepeda, R. E. (2009). Antibacterial and Antifungal Activities of Some Mexican Medicinal Plants. Journal of Medicinal Food, 12(6), 1398-1402. https://doi.org/10.1089/jmf.2008.0205

Sabandar, C. W., Ahmat, N., Jaafar, F. M., \& Sahidin, I. (2013). Medicinal property, phytochemistry and pharmacology of several Jatropha species (Euphorbiaceae): a review. Phytochemistry, 85, 7-29. https://doi.org/10.1016/j. phytochem.2012.10.009.

Tequida-Meneses, M., Cortez-Rocha, M., Rosas-Burgos, E. C., López-Sandoval, S. \& Corrales-Maldonado, C. (2002). Effect of alcoholic extracts of wild plants on the inhibition of growth of Aspergillus flavus, Aspergillus niger, Penicillium chrysogenum, Penicillium expansum, Fusarium moniliforme and Fusarium poae moulds. Revista Iberoamericana de Micología, 19(2), 84-88.

Terahara N., 2015. Flavonoids in Foods: A Review. Natural Product Communications. 10(3), 521-528. https://doi. org/10.1177/1934578X1501000334

Vega-Ruiz, Y. C., Hayano-Kanashiro, C., Gámez-Meza, N. \& Medina-Juárez, L.A. (2021). Determination of Chemical Constituents and Antioxidant Activities of Leaves and Stems from Jatropha cinerea (Ortega) Müll. Arg and Jatropha cordata (Ortega) Müll. Arg. Plants. 10, 212. https://doi. org $/ 10.3390 /$ plants 10020212

Wong-Paz, J. E., Contreras-Esquivel, J. C., RodríguezHerrera, R., Carrillo-Inungaray, M. L., López, L. I., Nevárez-Moorillón, G. V. \& Aguilar, C. N. (2015). Total phenolic content, in vitro antioxidant activity and chemical composition of plant extracts from semiarid Mexican region. Asian pacific journal of tropical medicine, 8(2), 104-111. https://doi.org/10.1016/S1995-7645(14)60299-6.

Xu D-P, Zhou Y, Zheng J, Li S, Li A-N. \& Li H-B. (2016). Optimization of Ultrasound-Assisted Extraction of Natural Antioxidants from the Flower of Jatropha integerrima by Response Surface Methodology. Molecules, 21(1), 18. https://doi.org/10.3390/molecules21010018.

Yetman, D. \& Van Devender, T. R., (2002). Mayo Ethnobotany. Land, History, and Traditional Knowledge in Northwest Mexico. University of California Press. https://doi. org/10.1525/9780520926356.

Zhang, X.-P., Zhang, M.-L., Su, X.-H., Huo, C.-H., Gu, Y.-C. \& Shi, Q.-W. (2009). Chemical Constituents of the Plants from Genus Jatropha. Chemistry \& Biodiversity, 6(12), 2166-2183. DOI : 10.1002/cbdv.200700461. 